Bol. Acad. peru. leng. 58. 2014 (111-127)

\title{
ITALIANISMOS EN LA VIGÉSIMA SEGUNDA EDICIÓN DEL DRAE
}

\section{ITALIANISMES DANS LA VINGT-DEUXIÈME ÉDITION DU DRAE}

\section{ITALIANISMS IN THE TWENTY-SECOND EDITION OF THE DICTIONARY OF THE SPANISH LANGUAGE OF THE ROYAL SPANISH ACADEMY OR DRAE}

\author{
Ana Baldoceda Espinoza \\ Universidad Nacional Mayor de San Marcos
}

\section{Resumen:}

En el presente artículo se examinan las palabras de origen italiano incorporadas en la 22. ${ }^{a}$ edición del DRAE, observando el tratamiento que reciben en dicho diccionario y mostrando las formas en que estos elementos léxicos se han asimilado al español, las adaptaciones y variaciones que han experimentado en ortografía y fonética.

Tenemos: a) palabras tomadas sin adaptación (malaria, piano, piloto); b) con diferente escritura o pronunciación en el Perú (brócoli, ciabatta, salame); c) falta de marca diatópica Perú (capo, coliseo, posta médica); d) italianismos que no figuran en el DRAE (panetón, menestrón, fuente de soda); e) falta de pronunciación figurada (carpaccio, crescendo, mezzosoprano); f) adaptación de sonidos italianos al pasar al español: $g n>\tilde{n}$ lasagna $>$ lasaña; infinitivos terminados en /e/ brillare $>$ brillar, en posición inicial de palabra o los grupos consonánticos $/ s p-, s f-, s k-/)$ pasan al español con 
un a /e/ protética: spaghetti > espagueti, sfumare > esfumar, scorzonera $>$ escorzonera.

\section{Résumé:}

Le présent article étudieles mots d'origine italienne incorporés dans

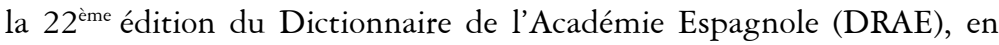
examinant le traitement qu'elles reçoivent dans ce dictionnaire et en montrant les procédés par lesquels ces éléments lexicaux se sont assimilés à l'espagnol, ainsi que les adaptations et variations qu'ils ont souffert dans l'orthographe et la phonétique.

Nous avons: a) des mots repris sans adaptation (malaria, piano, piloto); b) avec une écriture ou une prononciation différente au Pérou (brócoli, ciabatta, salame); c) manque de marque diatopique (capo, coliseo, posta médica); d) italianismes ne figurant pas sur le DRAE (panetón, menestrón, fuente de soda); e) manque de prononciation figurée (carpaccio, crescendo, mezzosoprano); f) adaptation de sons italiens en passant à l'espagnol: gn $>\tilde{n}$ lasagna $>$ lasaña; infinitifsfinissant par /e/ brillare $>$ brillar, groupe consonantiques $/ s p-$-, $s f-$, sk-/ en début de mot, qui passent à l'espagnol avec un /e/ d'appui: spaghetti > espagueti, sfumare > esfumar, scorzonera > escorzonera.

\section{Abstract:}

This article examines the words of Italian origin incorporated in the 22nd edition of DRAE, noting the treatment they receive in the dictionary and showing the ways how these lexical items have been assimilated into Spanish, the adaptations and variations they have undergone in spelling and pronunciation.

We have: a) words taken without adaptation (malaria, piano, piloto); b) with different spelling or pronunciation in Peru (broccoli, ciabatta, salami); c) lack of a diatopical mark in Peru (capo, coliseo, posta médica); d) Italianisms not listed in the DRAE (panetón, menestrón, fuente de soda); e) lack of figurative pronunciation (carpaccio, crescendo, mezzosoprano); f) adaptation of Italian sounds when entering into Spanish: $g n>\tilde{n}$ lasagna > lasagna; infinitives ending in /e/ brillare $>$ brillar in initial position of words or consonant groups /sp-, sf-, sk-/) enter into Spanish with a prosthetic /e/: spaghetti $>$ espaghetti, sfumare $>$ esfumar, scorzonera $>$ escorzonera. 
Palabras clave: Italianismos, DRAE, fonética, préstamos del italiano.

Mots clés: Italianismes, DRAE, phonétique, emprunts à l'italien.

Key words: Italianisms, DRAE, phonetics, Italian borrowings.

Fecha de recepción: $\quad$ 11/08/2014

Fecha de aceptación: $\quad$ 08/09/2014

En su desarrollo, el español es deudor del aporte de varias otras lenguas, como nativas peninsulares (vasco y hablas ibéricas extintas), lenguas románicas (principalmente portugués, francés e italiano) y lenguas amerindias, entre muchas otras.

De ellas, es la italiana la que ha dejado una mayor huella, pues desde el siglo XIv hasta la actualidad, ha aportado muchas palabras: en un tiempo por el prestigio de las letras y las artes de Italia; y en décadas recientes por la gastronomía.

Como corresponde a sus objetivos, el DRAE, en su 22. ${ }^{a}$ edición (2001), presenta numerosas palabras de origen italiano - que constituyen un total de 596 entradas - de las cuales buena parte se utilizan en el Perú, especialmente del campo de la gastronomía, la música, la arquitectura, la moda del vestido, la pintura, la navegación marina, etc.

A continuación presentamos cómo se dan los italianismos en el DRAE 22. ${ }^{a}$ ed.:

\section{a) Palabras sin adaptación}

Son vocablos que han ingresado al español, según el DRAE, sin ningún cambio en la grafía, tales como abate, adagio ${ }^{2}$, alabarda, altoparlante, anca, andante ${ }^{2}$, andantino, ándito, antitrinitario, 
ria, arabesco, aria, barcarola, barda ${ }^{1}$, bel canto, berlina ${ }^{2}$, bizarro, rra, bravata, camerino, camisola, cantábile, cantata, cantina, caparra $^{2}$, capo, caricato, caricatura, carmelita, carpaccio, carriola, casino, castrato, catafalco, cavatina, Cicerone, clavicémbalo, coda ${ }^{2}$, collarino, coloratura, comparsa, concertante, concertino, contralto, cordino, corno ${ }^{2}$, crescendo, descrescendo, dente, dintorno, divertimento, do ${ }^{1}$, dogaresa, druso, sa; dúo, fascismo, fermata, ferrovía, fiasco, filigrana, finta ${ }^{2}$, frasca ${ }^{1}$, floritura, fumarola, fusa, gamba, garbo, gorgonzola, granito ${ }^{2}$, gripo, guado, imposta, impronta, influenza, lava ${ }^{1}$, lena ${ }^{1}$, líbero, lira ${ }^{2}$, lontananza, madrépora, madrigalesco, mafia, mafioso(sa), magenta, malaria, malato, ta, mandorla, manierismo, mena ${ }^{3}$, mercadante, mescolanza, mezzosoprano, miniatura, moderato, mofeta, mordente, morra ${ }^{2}$, mozzarella, nepote, ocarina, orcina, organza, ossobuco, palco, paparazzi, pároli, partitura, pavana, pedante, pérgola, pernio, pianoforte, pian piano, piastra, piloto, pista, pizza, pizzicato, poema, pórfido, posta, premura, presto $^{2}$, prima donna,r ampollo, ravioli, reverso, romanza, rotonda, salami, saldo, saltimbanco, serenata, soda, sonata, sonatina, soprano, sorda, sorgo, taranta, tempo, tenorino, tómbolo, tondino, tondo, torso, trémolo, trío ${ }^{2}$, tromba, variopinto(ta), venturina, vibrato.

\section{b) Palabras con adaptación de tilde}

En italiano existen palabras con pronunciación esdrújula, pero en la escritura no se pone la marca ortográfica. Por ejemplo:

déspota. (Del it. despota).

góndola. (Del it. gondola).

ópera. (Del it. opera).

sémola. (Del it. semola)

tómbola. (Tomado del it. tómbola).

\section{Comentario}

En esta entrada no debe ser" (Tomado del it. tómbola)" sino "(Del it. tombola)", sin tilde. 
c) Diferente escritura o pronunciación de italianismos en el Perú

bróculi. (Del it. broccoli, y este de brocco). m. brécol. Comentario

En el Perú se pronuncia y escribe brócoli.

canelón ${ }^{3 .}$ (Del it.cannellone). m. Pasta alimenticia de harina en forma de lámina cuadrada con la que se envuelve un relleno. U. m. en pl.

\section{Comentario}

En el Perú no es la norma decir canelón, se utiliza solo en plural: canelones.

chapata. (Del it. ciabatta, y este de etim. disc.). f. Tipo de pan crujiente, de forma aplastada y alargada.

\section{Comentario}

En el Perú se escribe a la italiana, ciabatta, y se pronuncia /chabátal sin geminación de la /t/, como sí ocurre en italiano.

macarrón.(Del it. dialect. maccarone, y este del gr. $\mu \alpha \kappa \alpha \rho \omega ́ v \varepsilon ı \alpha$, canto mortuorio, comida funeraria). $\mathrm{m}$. Pasta alimenticia de harina que tiene forma de canuto más o menos alargado. U. m. en pl.

\section{Comentario}

En el Perú preferentemente se usa en plural: macarrones.

ossobuco. (Voz it.). m. Estofado de carne de vacuno, cortada del jarrete, con el hueso y su caña incluidos. $\| 2$. m. Arg. y Chile. Corte del hueso del jarrete vacuno, con su tuétano y la carne que lo rodea. Comentario

En Perú fluctúa el uso en la escritura entre ossobuco y osobuco, y se pronuncia /osobúko/.

raviole. (Del it. ravioli). m. ravioli. U. m. en pl. 


\section{Comentario}

No le corresponde llevar étimo.Debe quedar así, como remisión: "raviole. (De ravioli) m. ravioli. U. m. en pl."

ravioli. (Del it. ravioli). $\mathrm{m}$. Pasta alimenticia de harina, que se hace en forma de pequeños cuadrados rellenos de picadillo, verduras, requesón, etc. U. m. en pl.

\section{Comentario}

En el Perú predomina el plural y se escribe ravioles.

salami. (Del it. salami). m. Embutido hecho con carne vacuna y carne y grasa de cerdo, $[\ldots]$ se come crudo.

\section{Comentario}

En el Perú se pronuncia y escribe salame.

d) Italianismos en que falta la marca diatópica Perú

capo. (Del it. capo, cabeza, aplicado a los jefes de la mafia). m. Jefe de una mafia, especialmente de narcotraficantes. $\| 2 . \mathrm{m}$. coloq. Arg., Bol. y $U r$. jefe (\| superior de un cuerpo u oficio). $\| 3 . \mathrm{m}$. coloq. Arg., Bol., Par. y Ur. Persona con poder y prestigio o muy entendida en una determinada materia. Es un capo en física cuántica.

\section{Comentario}

En la acepción $\|$ 3. falta la marca Perú. También se utiliza la forma femenina capa y el aumentativo ponderativo capazote.

coliseo. (Del it. Colosseo, el famoso anfiteatro de Roma). m. Sala construida para espectáculos públicos. \|2. m. Bol., Ecuad. y Hond. Recinto cerrado para algunos juegos deportivos.

\section{Comentario}

En la segunda acepción falta la marca Perú.

libreto. (Del it. libretto). [...] ||2. m. Cuba y Ur. guion (\| texto en que se expone el contenido de un filme o de un programa de radio o televisión). 


\section{Comentario}

En la segunda acepción falta la marca Perú.

ossobuco. (Voz it.). [...] || 2. m. Arg. y Chile. Corte del hueso del jarrete vacuno, con su tuétano y la carne que lo rodea.

Comentario

En la segunda acepción falta la marca Perú.

pasquín. (Del it. Pasquino, nombre de una estatua en Roma, en la cual solían fijarse libelos o escritos satíricos). [...] ||2. m. El Salv., Nic., Ur. y Ven. Diario, semanario o revista con artículos e ilustraciones de mala calidad y de carácter sensacionalista y calumnioso. Comentario

En la segunda acepción falta la marca Perú.

posta. (Del it. posta). [...] $\| \sim$ médica. f. Cuba. Dispensario situado en una zona suburbana o rural.

Comentario

En la forma compleja $\| \sim$ médica. Falta la marca Perú.

ricota. (Del it. ricotta). f. Arg., Ur. y Ven. requesón.

Comentario

Falta la marca Perú.

rosticería. (Del it. rosticceria) f. Méx. y Nic. Establecimiento donde se asan y venden pollos.

Comentario

Falta la marca Perú.

tutilimundi. (Del it. tutti li mondi, todos los mundos). m. mundonuevo. $\|$ 2. m. Cuba y Méx. todo el mundo.

Comentario

Falta la marca Perú en la acepción $\| 2$. 
e) Italianismos que no figuran en el DRAE

\section{e.1 Propuestas deacepciones}

boleta. (Del it. bolletta). [...] 6. f. Arg., Ur. y Ven. Multa que se cobra a causa de una infracción de tránsito. Me bicieron la boleta. [...]

\section{Comentario}

Habría que agregar la acepción "Comprobante de pago" con la marca Perú.

casino. (Del it. casino, casa de campo). [...] || 4. m. Local donde, mediante pago, puede asistirse a espectáculos, conciertos, bailes y otras diversiones. Es propio de playas, balnearios, etc., y generalmente está destinado a la práctica de juegos de azar.[...]

\section{Comentario}

Falta la acepción naipe para Perú.

chaveta. (Del it. dialect. ciavetta, it. chiavetta). adj. coloq. loco (\| que ha perdido la razón). Se ha vuelto chaveta. [...] $\|$ 4. f. coloq. Cabeza humana. Hoy me duele la chaveta. \| 5. f. Cuba. Cuchilla pequeña y curva usada por tabaqueros y zapateros.

\section{Comentario}

Falta otra acepción, porque según Hildebrandt (2011: 77), chaveta "en el Perú y otros países americanos, designa una 'cuchilla filuda', con mango o sin él, usada por delincuentes chaveteros".

soda. (Del it. soda). [...] || 3. f. C. Rica. Establecimiento, por lo general más pequeño que un restaurante, donde se venden comidas y bebidas. [...].

\section{Comentario}

En el Perú la acepción \|| 3. se desarrolla como fuente de soda.

e.2 Propuestas de entradas. Por su conocida difusión, convendría incluir, entre otras, las siguientes palabras de origen italiano: 
avanti. (Del it. avanti). interj. Perú. U. para dar ánimo. Avanti, muchachos.

menestrón. (Del it. minestrone.) m. Perú. Sopa que se hace con carne de res, papa, queso, fideo, choclo, zanahoria, diversas verduras y albahaca.

ñoqui. (Del it. gnocchi pl. de gnocco). m. Perú. Bocado de pasta hecho de harina de papa o de sémola, que se come cocido y condimentado de diversas maneras.

panetón. (Del it. panettone) m. Perú. Bizcocho en forma cilíndrica y abombado en la parte superior, que contiene pasas y confituras en su interior, que se suele consumir en Fiestas Patrias, Navidad y Año Nuevo.

trattoria. (Del it.) f. Perú. Restaurante de comida italiana en que se expenden pastas.

f) Italianismos en cursivas. Según el DRAE se escriben así cuando su representación gráfica o su pronunciación son ajenas a las convenciones del español. En el tratamiento de este grupo de palabras, especialmente si son de uso limitado, una deficiencia del DRAE es la falta de pronunciación figurada, por ejemplo en:

adagio $^{2}$. (Voz it.) adv. m. Mús. Con movimiento lento. \| 2. m. Mús. Composición o parte de ella que se ha de ejecutar con este movimiento.

carpaccio. (Voz it.). m. Plato compuesto de lonchas de carne o pescado, cortadas muy finas y condimentadas con diversas especias, que se consume crudo.

crescendo. (Voz it., y esta del lat. crescendo, ger. de crescěre, crecer). 1. $\mathrm{m}$. Aumento gradual de la intensidad del sonido. $\| 2 . \mathrm{m}$. 
Aumento progresivo. $\|$ in $\sim$. loc. adv. Con aumento gradual. $E l$ enfado del público fue in crescendo.

mezzosoprano. (Voz it.). f. Voz media entre la de soprano y la de contralto. || 2. com. Persona que tiene voz de mezzosoprano.

mozzarella. (Voz it.). f. Queso de procedencia italiana, hecho originalmente con leche de búfala, que se come muy fresco.

ossobuco. (Voz it.). m. Estofado de carne de vacuno, cortada del jarrete, con el hueso y su caña incluidos. $\|$ 2. m. Arg. y Chile. Corte del hueso del jarrete vacuno, con su tuétano y la carne que lo rodea.

paparazzi. (Voz, del n. p. Paparazzo, un fotógrafo en el filme $L a$ dolce vita). m. pl. Fotógrafos de prensa que se dedican a hacer fotografías a los famosos sin su permiso.

pizza. (Voz it.). f. Especie de torta chata, hecha con harina de trigo amasada, encima de la cual se pone queso, tomate frito y otros ingredientes como anchoas, aceitunas, etc. Se cuece en el horno.

pizzicato. (Voz it.). adj. Mús. Dicho de un sonido: Que se obtiene en los instrumentos de arco pellizcando las cuerdas con los dedos. || 2. m. Mús. Trozo de música que se ejecuta de esta forma.

g) Adaptación de sonidos italianos al pasar al español. En este apartado vemos cómo han pasado al español, según registra el DRAE, algunos sonidos italianos, representados con las correspondientes grafías. Se observa en italiano que en muchos casos las consonantes geminan entre vocales, aunque esto no siempre se refleje en la escritura, por ejemplo, en lasagna /lazána/, que tiene consonante nasal palatal sonora.

- Palabras que en italiano se escriben con $<$ gli $>$ - cuyo sonido es lateral palatal, geminado entre vocales - han pasado al español 
escribiéndose con $<$ ll $>$, que representa dicha consonante lateral palatal sonora.

canalla. (Del it. canaglia).

folleto. (Del it. foglietto).

escollo. (Del it. scoglio).

medalla. (Del it. medaglia).

muralla. (Del it. muraglia, pared, muralla).

payaso, sa. (Del it. pagliaccio).

\section{Comentario}

Como corresponde con este grupo de palabras, pagliaccio debió dar pallaso, no payaso. Esto fue así porque el préstamo no se tomó directamente del italiano sino, a fines del siglo XVIII, del francés paillasse (Corominas, IV, 1985: 342), en una época en que la lateral palatal se había deslateralizado en francés y así llegó al español. pillar. (Del it. pigliare, coger). talla $^{3}$. (Del it. taglia, polea). tallarín. (Del it. tagliarini).

- El sonido/gn/ de italiano, geminado entre vocales, sale con /ñ/ en español, sin geminar:

bisoño, ña. (Del it. bisogno).

carroña. (Del it. carogna)

diseño. (Del it. disegno).

lasaña. (Del it. lasagna).

monseñor. (Del it. monsignore).

tacaño, ña. (Del it. taccagno).

- Formas del infinitivo en italiano pasan al castellano sin la $e$ final: acampar. (Del it. accampare).

brillar. (Del it. brillare).

charlar. (Del it. ciarlare).

comandar. (Del it. comandare).

cortejar. (Del it. corteggiare).

desfalcar. (Del it. defalcare)

desguazar. (Del it. sguazzare).

diseñar. (Del it. disegnare). 
festejar. (Del it. festeggiare). impostar. (Del it. impostare). intrigar. (Del it. intrigare). manejar. (Del it. maneggiare). regalar $^{1}$. (Cf. it. regalare).

\section{Comentario}

En el étimo no debe ir" (Cf. it.regalare)" sino "(Del it. regalare)". saldar. (Del it. saldare).

- Palabras en italiano que comienzan con /s/ se han incorporado en español con una /e/ protética:

esbelto, ta. (Del it. svelto).

esbirro. (Del it. sbirro).

escalinata. (Del it. scalinata).

escaramuza. (Del it. scaramuccia, combate breve y no decisivo).

escarcela. (Del it. scarsella, bolsa).

escarpín. (Del scarpino, dim. de scarpa, zapato).

escorzonera. (Del it. scorzonera).

esfumar. (Del it.sfumare).

espadachín. (Del it. spadaccino).

espagueti. (Del it. spaghetti).

estafeta. (Del it. staffetta).

estanza. (Del it. stanza).

estrafalario, ria. (Del it. dialect. strafalario, persona desaliñada). estropear. (Del it. stroppiare).

- Las palabras en italiano que contienen $<$ ll $>$ se pronuncian entre vocales con geminación y han pasado al español como $<\mathrm{l}>$, que representa una lateral alveolar sonora (simple, no alargada ni geminada):

alarma. (Del it. allarme).

alegro. (Del it. allegro).

bizcotela. (Del biscottella,dim. de biscotto).

boleta. (Del it. bolletta).

canelón ${ }^{3}$. (Del it.cannellone).

martelo. (Del it. martello, martillo). 
modelo. (Del it. modello).

mortadela. (Del it. mortadella).

novela. (Del it. novella, noticia, relato novelesco).

pasarela. (Del it. passerella).

- Entre las vocales /a/ y /o/ la doble $\langle\mathrm{cc}\rangle$ del italiano se pronuncia con geminación $(/ \mathrm{kk} /)$, que en español se interpreta como $/ \mathrm{k} /$ simple:

acampar. (Del it. accampare).

bicoca. (Del it. bicocca, y este de Bicocca, población italiana al oeste de Milán, y nombre de la batalla que en este lugar libraron franceses y españoles en 1522).

brocado, da. (Del broccato, y este de brocco).

bróculi. (Del broccoli, y este de brocco).

macarrón. (Del it. dialect. maccarone).

morlaco ${ }^{1}$, ca. (Del it. morlacco, hombre rústico).

tacaño, ña. (Del it. taccagno).

tocata $^{1}$. (Del it. toccata).

- En italiano, en inicio de palabra o sílaba, las letras <ci $>$ representan una africada palatal sorda. Cuando en italiano tenemos $<c c i>$, en esta grafía compuesta la primera $\langle c\rangle$ representa una oclusiva sorda en posición final de sílaba, y las letras que siguen $(<\mathrm{ci}\rangle)$ corresponden a una africada palatal sorda (así, capriccio se pronuncia /kaprítčo/ y en español /kapríčo/; carpaccio, /karpátčo/ y en español /karpáčo/ ). Tenemos:

capricho. (Del it. capriccio).

carpaccio. (Voz it.).

cartucho. (Del it. cartoccio).

cucurucho. (Del it. dialect. cucuruccio).

chao. (Del it. ciao).

chapata. (Del it. ciabatta, y este de etim. disc.).

charlar. (Del it. ciarlare).

charlatán, na. (Del it. ciarlatano).

chaveta. (Del it. dialect. ciavetta, it. chiavetta). 
- En italiano la secuencia <ggi> se pronuncia como africada palatal sonora; al pasar al español se realiza como una fricativa velar sorda $/ \mathrm{x} /$ :

agio. (Del it. aggio).

arpegio. (Del it. arpeggio).

cortejar. (Del it. corteggiare).

cortejo. (Del it. corteggio).

festejar. (Del it. festeggiare).

logia. (Del it. loggia).

manejar. (Del it. maneggiare).

reja $^{2}$. (Del it. reggia, y este del lat. [porta] reğ̌a).

- Palabras con $<\mathrm{tt}>$ en italiano representan geminación de la consonante; en español no existe geminación ni se escribe $<$ tt $>$ sino $<\mathrm{t}>$ :

alegreto. (Del it. allegretto)

bancarrota. (Del it. bancarotta).

batuta. (Del it. battuta, pulsación).

boleta. (Del it. bolletta).

cancioneta. (Del it. canzonetta).

confeti. (Del it. confetti, confites).

cuarteta. (Del it. quartetta).

cuarteto. (Del it. quartetto).

cuatrocientos. (Del it. quattrocento).

diletante. (Del it. dilettante, que se deleita).

dueto. (Del it. duetto).

espagueti. (Del it. spaghetti).

estafeta. (Del it. staffetta).

gaceta $^{1}$. (Del it. gazzetta).

gaveta. (Del it. gavetta).

gueto. (Del it. ghetto).

maqueta. (Del it. macchietta).

minueto. (Del it. minuetto).

opereta. (Del it. operetta).

peto. (Del it. petto, pecho, y este del lat. pectus, pecho).

piñata. (Del it. pignatta). 
quinteto. (Del it. quintetto).

ricota. (Del it. ricotta).

soneto. (Del it. sonetto, y este del lat. sonus, sonido).

- En italiano la doble /ff/ se gemina, es decir, se produce alargamiento; mientras que en español no ocurre aquello ni se escribe duplicando la letra.

estafa $^{2}$. (Del it. staffa, estribo).

estafeta. (Del it. staffetta).

grafito $^{2}$. (Del it. graffito).

jirafa. (Del it. giraffa).

- En italiano <ss $>$ indica geminación de /s/; en español ni se alarga la consonante ni se pone doble letra:

asalto. (Del it. assalto).

foso. (Del it. fosso).

fracasar. (Cf. it. fracassare).

Comentario

En el étimo no debe ir "(Cf. it. fracassare)", sino "(Del it. fracassare)". pasarela. (Del it. passerella).

tesitura. (Del it. tessitura).

- En italiano palabras con doble $<p p>$ indican geminación, que en español no ocurre fonéticamente ni en la escritura:

apartamento. (Del it. appartamento y este del esp. apartamiento, con reducción de diptongo).

capuchino, na. (Del it. cappuccino).

estropear. (Del it. stroppiare).

grupo. (Del it. gruppo).

- Determinadas palabras que en italiano empiezan con /i/ en español se hace /e/:

embasamiento. (Del it. imbasamento).

embecadura. (Del it. imbeccare, meter en el pico).

empatar. (Del it. impattare, terminar iguales, sin ganar ni perder). empresa. (Del it. impresa). 


\section{Conclusiones}

Con la finalidad de enriquecer en el DRAE el tratamiento de los préstamos del italiano, sugerimos tomar en cuenta lo siguiente:

1) Conviene que el DRAE ponga la pronunciación en los italianismos crudos como adagio, carpaccio, etc.

2) En el espacio del étimo no deben poner "(Cf.)", como en "fracasar. (Cf. it. fracassare)","regalar ${ }^{1}$, (Cf. it. regalare)"; ni "Tomado del" como en "tómbola. (Tomado del it. tómbola)". Deben dar un solo tratamiento, y los ejemplos mencionados quedarían así: "fracasar. "(Del. it. fracassare)", "regalar ${ }^{1}$, (Del. it. regalare)", "tómbola. (Del it. tombola)", cuyo étimo no debe llevar tilde.

3) En la entrada "raviole. (Del it. ravioli). [...]" no se debe poner étimo; debe quedar así: "raviole. (De ravioli) m. ravioli. U. m. en pl."

4) Se necesita incluir en la próxima edición del DRAE la marca Perú en acepciones de palabras como capo, pasquín, libreto, etc.; y nuevas acepciones como 'naipe' en casino, 'comprobante de pago' en boleta, etc.

5) Como propuestas de nuevas entradas tendríamos avanti, ñoqui, panetón, menestrón y trattoria, entre otras. 


\section{BIBLIOGRAFÍA}

REAL ACADEMIA ESPAÑOLA. Diccionario de la Lengua Española. Madrid, Espasa-Calpe S. A., 22. edición, 2001.

ÁlVAREZ VITA, Juan. Diccionario de Peruanismos. Lima, Universidad Alas Peruanas, 2. ${ }^{a}$ edición, 2009.

COROMINAS, Juan y Pascual, J. A. Diccionario crítico etimológico Castellano e Hispánico (tomo III). Madrid, Gredos, 1. ${ }^{a}$ reimpresión de la 1. aedición, 1985.

HILDEBRANDT, Martha. 1000 palabras y frases peruanas. BarcelonaLima, Espasa Libros S. L. U., 2011.

HYPERPOLYGLOTTE.COM. "Guía de pronunciación del italiano". En http://hyperpolyglotte.com/aprende_italiano/pron_guia.php. En Lima 21_03-2011 a las 17:03 h.

LAPESA, Rafael. Historia de la lengua española. Madrid, Gredos S. A. 8. ${ }^{a}$ edición, 2008.

TAM, Laura. Diccionario Español-Italiano/Italiano-Spagnolo. Milán, Grupo Océano, 2009.

\section{Correspondencia:}

\section{Ana Baldoceda Espinoza}

Docente del Departamento Académico de Lingüística de la Facultad de Letras y Ciencias Humanas de la Universidad Nacional Mayor de San Marcos. Correo electrónico: anabal_2000@yahoo.com.pe 